\title{
Diversity of the RNA Polymerase in the H7N9 Influenza A Virus
}

\author{
Jing Li, Wenjun Liu* \\ CAS Key Laboratory of Pathogenic Microbiology and Immunology, Institute of Microbiology, Chinese Academy \\ of Sciences, Beijing, China \\ Email: lj418@163.com,
}

Received 20 July 2015; accepted 24 August 2015; published 27 August 2015

Copyright (C) 2015 by authors and Scientific Research Publishing Inc.

This work is licensed under the Creative Commons Attribution International License (CC BY).

http://creativecommons.org/licenses/by/4.0/

c) (i) Open Access

\section{Abstract}

A novel influenza virus of the H7N9 subtype has infected more than 350 people in China since 19 February 2013. Evolutionary analysis indicates that the virus is a reassortant originated from $\mathrm{H7}$, $\mathrm{N} 9$ and H9N2 avian influenza viruses, and bears some amino acids associated with mammalian receptor binding, raising concern over the possibility of a new influenza pandemic. Besides HA and NA, the mutation of the polymerase is known to have an important role in virulence, host adaptation and transmissibility in mammalians. In this article, the annotation of the polymerase protein domain associated with molecular function has been highlighted, suggesting the combination of RNA polymerase of $\mathrm{H7N} 9$ viruses is still not stable for host adaptation. In addition, the mutation hallmarks in polymerase gene of $\mathrm{H} 7 \mathrm{~N} 9$ are compared, providing the potential determinants of the evolution in the $\mathrm{H} 7 \mathrm{~N} 9$ influenza A virus.

\section{Keywords}

Diversity, RNA Polymerase, H7N9 Influenza A Virus

Since Feb 19, 2013, when the initial patient infected with the novel influenza A H7N9 virus from an avian source showed symptoms, 350 laboratory-confirmed cases including more than 50 deaths had been reported in mainland China as of June 8, 2015 (http://www.who.int/csr/don/2013 08 11/en/index.html). After nearly 70 days' quiescence, the cases of human infection with H7N9 virus re-emerged in Zhejiang and Guangdong Province [1]. This virus seems to exhibit low pathogenicity in birds, by contrast with the severe disease that occurs in human beings [2]. Another notable feature of H7N9 is the rapid accumulation of laboratory-confirmed cases of infection in human being, even though phylogenetic and epidemiological evidence suggests that transmission is mainly zoonotic [3]-[7]. This has raised global concerns regarding this novel avian-origin AIV.

Based on phylogenetic analysis of isolates from human and domestic birds, it has been proposed that the ${ }^{*}$ Corresponding author. 
H7N9 viruses responsible for this outbreak are novel reassortants. Their haemagglutinin (HA) and neuraminidase (NA) genes probably originated from Eurasian avian influenza viruses; the internal genes are derived from avian H9N2 viruses that circulated recently in China [8] [9]. The influenza virus polymerase complex, which is a heterotrimer formed by the PB1, PB2, and PA subunits, is a major determinant of species specificity, transmission, and pathogenesis [10] [11]. In particular, three genetic substitutions K627E, N701D, and R591Q in the PB2 segment of influenza virus have been reported to affect host cell tropism [12]-[14]. A single substitution of glutamate to lysine at the residue 627 (E627K) facilitates the adaption of H5N1 and other avian influenza viruses to mammals and increases transmission and pathogenesis in humans, mice, ferrets, and guinea pigs [14] [15].

To address the possibility that the novel H7N9 virus can better adapt to humans, we analyzed and compared the genomes of H7N9 viruses isolated form fatal cases with those of relevant H7N9 viruses. We particularly focus on the polymerase genes which are more determinant to over the host adaptation and the species barriers.

The genome gene sequence of A/Shanghai/1/2013 was used as a reference sequence to compute the amino acid identities for other polymerase gene sequences. It is observed that other 20 strains all display the highest similarity value. The median identity is range from $98.3 \%$ to $98.6 \%$ (referred as PB1, PB2 and PA).

Of the three P proteins, PB1 is the best characterized functionally. Biochemical and structural analyses recognize PB1 as responsible for RNA chain elongation [16]. PB1 contains amino acid motifs common to all RNAdependent RNA polymerases and RNA-dependent DNA polymerases [17]. Mutations within these motifs render the complex inactive for transcription and replication in tissue culture cells [18] [19]. The previous study has showed that PB1 I368V is correlated with H5N1 virus airborne transmission among ferrets [20] [21]. In accordance with this deduction, except Shanghai/1 and Environment/Wuxi/1, all 19 strains H7N9 viruses analyzed here encode PB1-368V. Aside from this position, the residue I to V mutation also found in 164, 528, 637 and 728 positions. PB1-F2, which is encoded on a +1 reading frame of the gene segment for influenza viral RNA polymerase subunit PB1, is a known virulence factor. Specifically, an asparagine-to-serine substitution at position 66 (N66S), which is found in 1918 pandemic viruses, is partly responsible for the high pathogenicity of this virus. In contrast, all H7N9 viruses lack the N66S mutation [20]. In addition, according to the sequence analysis, we found that almost all human H7N9 viruses encoded a full length PB1-F2 of 90 amino acids except the A/Pigeon/shanghai/S1069/2013 virus, which was truncated by a stop codon at position 26. Previous research had shown that truncation the PB1-F2 protein had no effect on viral replication in tissue culture but diminished virus pathogenicity and mortality in mice. Taking it into account, the A/Pigeon/Shanghai/S1069/2013 virus which isolated from a pigeon collecting on 2 and 3 April from a Shanghai market, may be have potentially to increase the risk to mammalian animals. It is noted that A/Pigeon/Shanghai/S1069/2013 virus also have two characteristic substitution, including 48 from $\mathrm{R}$ to $\mathrm{Q}$ and 53 from $\mathrm{K}$ to $\mathrm{R}$.

Amino acid 627 of the PB2 protein is almost exclusively a lysine in human influenza virus isolates and a glutamic acid in avian influenza strains [14]. This residue was first identified as a determinant of host range in 1993, and was later shown to contribute to the pathogenicity and transmission in mammalian hosts in H5N1 and H7N7 subtype influenza virus infection, when the residue substituted from E to K [12] [15] [22]. Interestingly, PB2-627K is rare among avian H9N2 PB2 proteins [9]. In keeping with this finding, the avian and environment H7N9 influenza viruses encode PB2-627E. By contrast, most human H7N9 viruses encode PB2-627K. Only A/Shanghai/4/2013, A/Zhejiang/2/2013 and A/Zhejiang/DTID-ZJU01/2013 maintain the residue with PB2-627E. Different from the other H7N9 virus, the A/Zhejiang/2/2013 and A/Zhejiang/DTID-ZJU01/2013 virus PB2 have D701N instead of E627K, which can reportedly compensate for the lack of $627 \mathrm{~K}$ in terms of increasing transmission, as well as enhancing the virus replication. Similarly to E627K, a change of PB2 amino acid 701 from aspartic acid to asparagines has been implicated in expanding the host range. The PA protein, which has function in both transcription and replication, has been reported to contribute to the regulation of the host response upon viral infection or increase the virulence of a low-pathogenic avian H5N2 influenza virus [23]-[25]. Interestingly, compared with the genome sequence, we found the characteristic mutation at position 308, except A/Hangzhou/3/2013 and A/Environment/Wuxi/1/2013 viruses, almost all viruses showed 308I, the other two viruses had $308 \mathrm{~V}$. Previous study had shown that the A/Wuxi/1/2013 and A/Wuxi/2/2013 were able to transmit from person to person [26], though the limited and non-sustainable transmissibility, and demonstrated that the mutation of I308V may contribute to increasing the viral transmission.

The pathogenesis and transmissibility of influenza viruses is a polygenic trait wherein molecular determinants may differ among animal species [27]. The main mechanisms that facilitate the acquisition of virulence factors among influenza viruses are mutations due to faulty viral RNA replication and genetic reassortment with cocir- 
culating influenza viruses [28] [29]. The polymerase subunits were responsible for the transcription and replication of the viral RNA genome in the nuclei of infected cells. Efficient RNA polymerase inducing high speed of virus growth can out-compete the antiviral response of the infected host cells. Notably, each of the previous 20th-century influenza virus pandemic strains has included a novel PB1 in addition the new HA and/or NA segment. Furthermore, the compatibility of the polymerase subunits may be a restricting factor for influenza virus reassortment [30]. Therefore, we should highlight the pivotal role of the viral polymerases in the evolution of influenza viruses with respect to reassortment and the production of new, more-virulent pandemic viruses (Figure 1, Table 1).

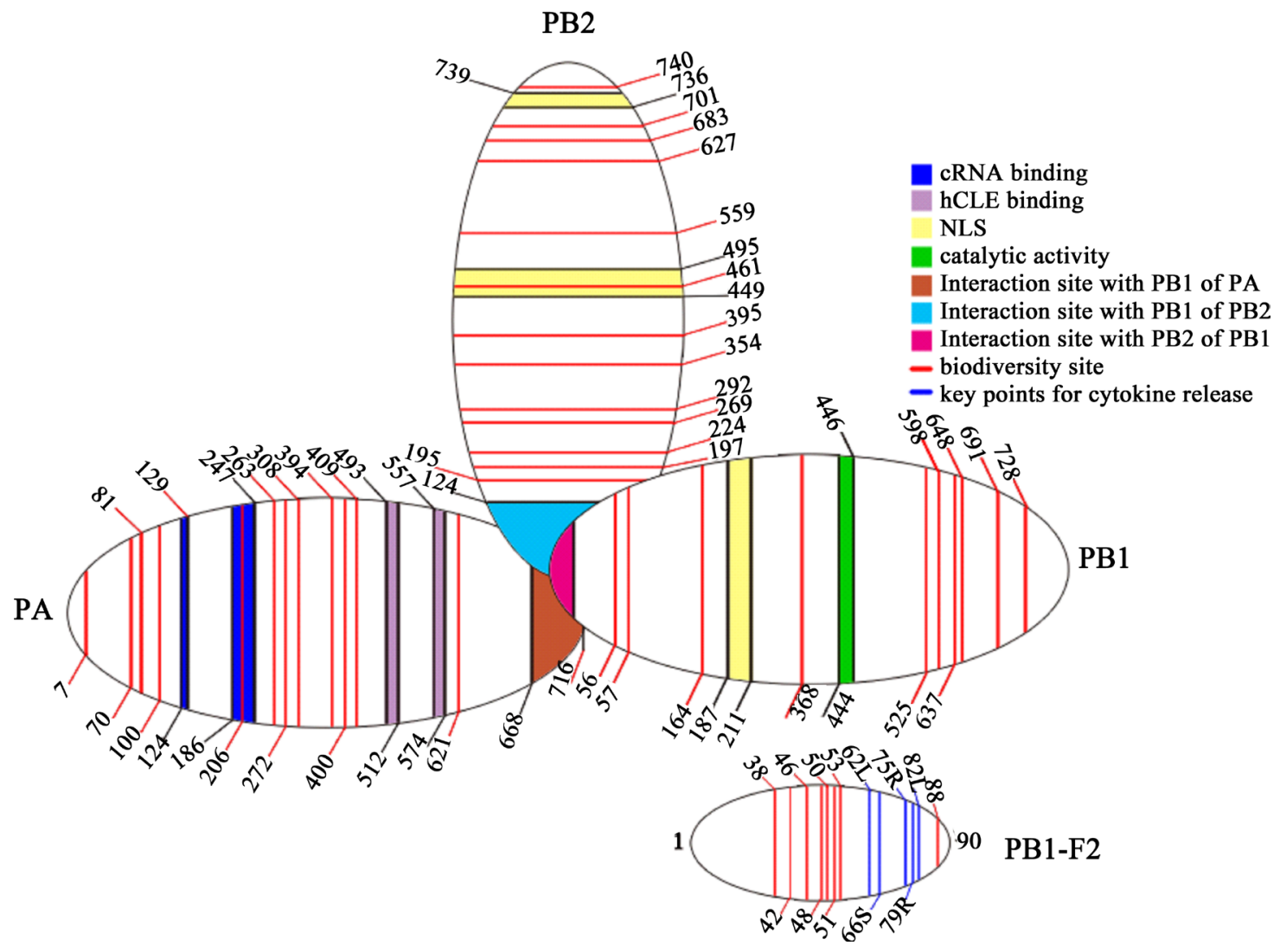

Figure 1. Diversity of the polymerase proteins of the H7N9 Influenza A Virus.

Table 1. Identification of differential amino acids of RNA polymerase in the H7N9 influenza A virus.

\begin{tabular}{cccccccccccc}
\hline & & & \multicolumn{3}{c}{ PB1 } & & & \\
& & 56 & 57 & 164 & 368 & 528 & 598 & 637 & 648 & 691 & 728 \\
\hline A/Shanghai/1/2013 & $\mathrm{T}$ & $\mathrm{T}$ & $\mathrm{I}$ & $\mathrm{I}$ & $\mathrm{V}$ & $\mathrm{M}$ & $\mathrm{V}$ & $\mathrm{A}$ & $\mathrm{K}$ & $\mathrm{I}$ \\
A/Shanghai/2/2013 & $\mathrm{T}$ & $\mathrm{T}$ & $\mathrm{I}$ & $\mathrm{V}$ & $\mathrm{V}$ & $\mathrm{L}$ & $\mathrm{I}$ & $\mathrm{A}$ & $\mathrm{K}$ & $\mathrm{I}$ \\
A/Shanghai/3/2013 & $\mathrm{T}$ & $\mathrm{T}$ & $\mathrm{I}$ & $\mathrm{V}$ & $\mathrm{V}$ & $\mathrm{L}$ & $\mathrm{I}$ & $\mathrm{A}$ & $\mathrm{K}$ & $\mathrm{I}$ \\
A/Shanghai/4/2013 & $\mathrm{T}$ & $\mathrm{T}$ & $\mathrm{I}$ & $\mathrm{V}$ & $\mathrm{V}$ & $\mathrm{L}$ & $\mathrm{I}$ & $\mathrm{A}$ & $\mathrm{K}$ & $\mathrm{I}$ \\
A/Shanghai/4664T/2013 & $\mathrm{T}$ & $\mathrm{T}$ & $\mathrm{I}$ & $\mathrm{V}$ & $\mathrm{V}$ & $\mathrm{L}$ & $\mathrm{I}$ & $\mathrm{A}$ & $\mathrm{K}$ & $\mathrm{I}$ \\
A/Chicken/shanghai/S1053/2013 & $\mathrm{T}$ & $\mathrm{T}$ & $\mathrm{I}$ & $\mathrm{V}$ & $\mathrm{V}$ & $\mathrm{L}$ & $\mathrm{I}$ & $\mathrm{A}$ & $\mathrm{K}$ & $\mathrm{I}$ \\
A/Pigeon/shanghai/S1069/2013 & $\mathrm{A}$ & $\mathrm{M}$ & $\mathrm{I}$ & $\mathrm{V}$ & $\mathrm{I}$ & $\mathrm{L}$ & $\mathrm{I}$ & $\mathrm{S}$ & $\mathrm{K}$ & $\mathrm{I}$ \\
A/Environment/shanghai/S1088/2013 & $\mathrm{T}$ & $\mathrm{T}$ & $\mathrm{I}$ & $\mathrm{V}$ & $\mathrm{V}$ & $\mathrm{L}$ & $\mathrm{I}$ & $\mathrm{A}$ & $\mathrm{K}$ & $\mathrm{I}$ \\
A/Zhejiang/01/2013 & $\mathrm{T}$ & $\mathrm{T}$ & $\mathrm{I}$ & $\mathrm{V}$ & $\mathrm{V}$ & $\mathrm{L}$ & $\mathrm{I}$ & $\mathrm{A}$ & $\mathrm{K}$ & $\mathrm{I}$ \\
A/Zhejiang/1/2013 & $\mathrm{T}$ & $\mathrm{T}$ & $\mathrm{I}$ & $\mathrm{V}$ & $\mathrm{V}$ & $\mathrm{L}$ & $\mathrm{I}$ & $\mathrm{A}$ & $\mathrm{K}$ & $\mathrm{I}$ \\
\hline
\end{tabular}




\section{Continued}

\begin{tabular}{|c|c|c|c|c|c|c|c|c|c|c|}
\hline A/Zhejiang/2/2013 & $\mathrm{T}$ & $\mathrm{T}$ & I & $\mathrm{V}$ & $\mathrm{V}$ & $\mathrm{L}$ & I & A & $\mathrm{K}$ & I \\
\hline A/Zhejiang/DTID-ZJU01/2013 & $\mathrm{T}$ & $\mathrm{T}$ & I & $\mathrm{V}$ & $\mathrm{V}$ & $\mathrm{L}$ & I & A & K & I \\
\hline A/Chicken/zhejiang/DTID-ZJU01/2013 & $\mathrm{T}$ & $\mathrm{T}$ & I & $\mathrm{V}$ & $\mathrm{V}$ & $\mathrm{L}$ & I & A & K & I \\
\hline A/Hangzhou/1/2013 & $\mathrm{T}$ & $\mathrm{T}$ & I & V & $\mathrm{V}$ & $\mathrm{L}$ & I & A & K & I \\
\hline A/Hangzhou/2/2013 & $\mathrm{T}$ & $\mathrm{T}$ & I & $\mathrm{V}$ & $\mathrm{V}$ & $\mathrm{L}$ & I & A & K & $\mathrm{V}$ \\
\hline A/Hangzhou/3/2013 & $\mathrm{T}$ & $\mathrm{T}$ & $\mathrm{V}$ & V & I & $\mathrm{L}$ & I & A & K & I \\
\hline A/Environment/Hangzhou/34/2013 & $\mathrm{T}$ & $\mathrm{T}$ & I & $\mathrm{V}$ & V & $\mathrm{L}$ & I & A & K & I \\
\hline A/Anhui/1/2013 & $\mathrm{T}$ & $\mathrm{T}$ & I & V & $\mathrm{V}$ & $\mathrm{L}$ & I & A & K & I \\
\hline A/Wuxi/1/2013 & $\mathrm{T}$ & $\mathrm{T}$ & I & $\mathrm{V}$ & V & $\mathrm{L}$ & I & A & K & I \\
\hline A/Wuxi/2/2013 & $\mathrm{T}$ & $\mathrm{T}$ & I & V & V & $\mathrm{L}$ & I & A & $\mathrm{R}$ & I \\
\hline A/Environment/Wuxi/1/2013 & $\mathrm{T}$ & $\mathrm{T}$ & I & I & $\mathrm{V}$ & $\mathrm{L}$ & $\mathrm{F}$ & A & K & I \\
\hline
\end{tabular}

\begin{tabular}{|c|c|c|c|c|c|c|c|}
\hline & \multicolumn{7}{|c|}{ PB1-F2 } \\
\hline & $1-38$ & 42 & 46 & 48 & 50 & 51 & 53 \\
\hline A/Shanghai/1/2013 & No deletion & $\mathrm{C}$ & $\mathrm{T}$ & $\mathrm{R}$ & G & $\mathrm{T}$ & $\mathrm{K}$ \\
\hline A/Shanghai/2/2013 & No deletion & $\mathrm{Y}$ & $\mathrm{T}$ & $\mathrm{R}$ & G & M & $\mathrm{K}$ \\
\hline A/Shanghai/3/2013 & No deletion & $\mathrm{Y}$ & $\mathrm{T}$ & $\mathrm{R}$ & G & M & $\mathrm{K}$ \\
\hline A/Shanghai/4/2013 & No deletion & $\mathrm{Y}$ & $\mathrm{T}$ & $\mathrm{R}$ & G & M & $\mathrm{K}$ \\
\hline A/Chicken/shanghai/S1053/2013 & No deletion & $\mathrm{Y}$ & $\mathrm{T}$ & $\mathrm{R}$ & G & M & $\mathrm{K}$ \\
\hline A/Pigeon/shanghai/S1069/2013 & Deletion & $\mathrm{C}$ & $\mathrm{T}$ & Q & $\mathrm{D}$ & M & $\mathrm{R}$ \\
\hline A/Environment/shanghai/S1088/2013 & No deletion & $\mathrm{Y}$ & $\mathrm{T}$ & $\mathrm{R}$ & G & M & $\mathrm{K}$ \\
\hline A/Zhejiang/01/2013 & No deletion & $\mathrm{Y}$ & $\mathrm{T}$ & $\mathrm{R}$ & G & M & $\mathrm{K}$ \\
\hline A/Zhejiang/1/2013 & No deletion & $\mathrm{Y}$ & $\mathrm{T}$ & $\mathrm{R}$ & G & M & $\mathrm{K}$ \\
\hline A/Zhejiang/2/2013 & No deletion & $\mathrm{Y}$ & $\mathrm{T}$ & $\mathrm{R}$ & G & M & $\mathrm{K}$ \\
\hline A/Hangzhou/1/2013 & No deletion & $\mathrm{Y}$ & $\mathrm{T}$ & $\mathrm{R}$ & G & M & $\mathrm{K}$ \\
\hline A/Hangzhou/2/2013 & No deletion & $\mathrm{Y}$ & $\mathrm{T}$ & $\mathrm{R}$ & G & M & $\mathrm{K}$ \\
\hline A/Hangzhou/3/2013 & No deletion & $\mathrm{C}$ & M & $\mathrm{R}$ & $\mathrm{D}$ & M & $\mathrm{K}$ \\
\hline A/Environment/Hangzhou/34/2013 & No deletion & Y & $\mathrm{T}$ & $\mathrm{R}$ & G & M & $\mathrm{K}$ \\
\hline A/Anhui/1/2013 & No deletion & $\mathrm{Y}$ & $\mathrm{T}$ & $\mathrm{R}$ & G & M & $\mathrm{K}$ \\
\hline A/Wuxi/1/2013 & No deletion & $\mathrm{Y}$ & $\mathrm{T}$ & $\mathrm{R}$ & G & M & $\mathrm{K}$ \\
\hline A/Wuxi/2/2013 & No deletion & $\mathrm{Y}$ & $\mathrm{T}$ & $\mathrm{R}$ & G & M & $\mathrm{K}$ \\
\hline A/Environment/Wuxi/1/2013 & No deletion & $\mathrm{C}$ & $\mathrm{T}$ & $\mathrm{R}$ & G & $\mathrm{T}$ & $\mathrm{K}$ \\
\hline
\end{tabular}

\begin{tabular}{|c|c|c|c|c|c|c|c|c|c|c|c|c|}
\hline & \multicolumn{12}{|c|}{ PB2 } \\
\hline & 195 & 197 & 224 & 292 & 354 & 395 & 461 & 559 & 627 & 682 & 701 & 740 \\
\hline A/Shanghai/1/2013 & $\mathrm{D}$ & $\mathrm{K}$ & $\mathrm{T}$ & $\mathrm{V}$ & I & S & $\mathrm{V}$ & $\mathrm{T}$ & $\mathrm{K}$ & G & $\mathrm{D}$ & $\mathrm{D}$ \\
\hline A/Shanghai/2/2013 & $\mathrm{D}$ & K & $\mathrm{T}$ & I & I & A & I & $\mathrm{N}$ & $\mathrm{K}$ & G & $\mathrm{D}$ & $\mathrm{D}$ \\
\hline A/Shanghai/3/2013 & $\mathrm{D}$ & K & $\mathrm{T}$ & V & $\mathrm{V}$ & A & I & $\mathrm{N}$ & $\mathrm{K}$ & G & $\mathrm{D}$ & $\mathrm{D}$ \\
\hline A/Shanghai/4/2013 & $\mathrm{D}$ & $\mathrm{K}$ & $\mathrm{T}$ & V & I & A & I & $\mathrm{N}$ & $\mathrm{E}$ & G & $\mathrm{D}$ & $\mathrm{D}$ \\
\hline A/Shanghai/4664T/2013 & $\mathrm{D}$ & $\mathrm{K}$ & $\mathrm{T}$ & V & $\mathrm{V}$ & A & I & $\mathrm{N}$ & $\mathrm{K}$ & G & $\mathrm{D}$ & $\mathrm{D}$ \\
\hline A/Chicken/shanghai/S1053/2013 & $\mathrm{D}$ & K & $\mathrm{T}$ & V & I & A & I & $\mathrm{N}$ & $\mathrm{E}$ & G & $\mathrm{D}$ & $\mathrm{D}$ \\
\hline A/Pigeon/shanghai/S1069/2013 & $\mathrm{D}$ & $\mathrm{R}$ & $\mathrm{T}$ & V & I & A & I & $\mathrm{N}$ & $\mathrm{E}$ & G & $\mathrm{D}$ & $\mathrm{D}$ \\
\hline A/Environment/shanghai/S1088/2013 & $\mathrm{D}$ & K & $\mathrm{T}$ & V & I & A & I & $\mathrm{N}$ & $\mathrm{E}$ & G & $\mathrm{D}$ & $\mathrm{D}$ \\
\hline A/Zhejiang/01/2013 & $\mathrm{D}$ & K & $\mathrm{S}$ & $\mathrm{V}$ & I & A & I & $\mathrm{N}$ & $\mathrm{K}$ & G & $\mathrm{D}$ & $\mathrm{D}$ \\
\hline
\end{tabular}




\section{Continued}

\begin{tabular}{|c|c|c|c|c|c|c|c|c|c|c|c|c|c|}
\hline \multicolumn{2}{|l|}{ A/Zhejiang/1/2013 } & $\mathrm{D}$ & $\mathrm{K}$ & S & $\mathrm{V}$ & I & A & I & $\mathrm{N}$ & $\mathrm{K}$ & $\mathrm{X}$ & $\mathrm{D}$ & $\mathrm{D}$ \\
\hline \multicolumn{2}{|l|}{ A/Zhejiang/2/2013 } & $\mathrm{D}$ & K & $\mathrm{T}$ & $\mathrm{V}$ & I & A & I & $\mathrm{N}$ & $\mathrm{E}$ & G & $\mathrm{N}$ & $\mathrm{D}$ \\
\hline \multicolumn{2}{|c|}{ A/Zhejiang/DTID-ZJU01/2013 } & $\mathrm{D}$ & K & $\mathrm{T}$ & $\mathrm{V}$ & I & $\mathrm{S}$ & I & $\mathrm{N}$ & $\mathrm{E}$ & G & $\mathrm{N}$ & $\mathrm{D}$ \\
\hline \multicolumn{2}{|c|}{ A/Chicken/zhejiang/DTID-ZJU01/2013 } & $\mathrm{D}$ & $\mathrm{K}$ & $\mathrm{T}$ & $\mathrm{V}$ & I & A & I & $\mathrm{N}$ & $\mathrm{E}$ & G & $\mathrm{D}$ & $\mathrm{D}$ \\
\hline \multicolumn{2}{|c|}{ A/Hangzhou/1/2013 } & $\mathrm{D}$ & $\mathrm{K}$ & S & $\mathrm{V}$ & I & A & I & $\mathrm{N}$ & K & G & $\mathrm{D}$ & $\mathrm{D}$ \\
\hline \multicolumn{2}{|c|}{ A/Hangzhou/2/2013 } & $\mathrm{E}$ & $\mathrm{R}$ & $\mathrm{T}$ & $\mathrm{V}$ & I & A & I & $\mathrm{T}$ & $\mathrm{K}$ & G & $\mathrm{D}$ & $\mathrm{D}$ \\
\hline \multicolumn{2}{|c|}{ A/Hangzhou/3/2013 } & $\mathrm{D}$ & $\mathrm{K}$ & $\mathrm{T}$ & $\mathrm{V}$ & I & A & I & $\mathrm{N}$ & $\mathrm{K}$ & G & $\mathrm{D}$ & A \\
\hline \multicolumn{2}{|c|}{ A/Environment/Hangzhou/34/2013 } & $\mathrm{D}$ & $\mathrm{K}$ & $\mathrm{T}$ & $\mathrm{V}$ & I & A & I & $\mathrm{N}$ & E & G & $\mathrm{D}$ & $\mathrm{D}$ \\
\hline \multicolumn{2}{|l|}{ A/Anhui/1/2013 } & $\mathrm{D}$ & $\mathrm{K}$ & $\mathrm{T}$ & $\mathrm{V}$ & I & A & I & $\mathrm{N}$ & $\mathrm{K}$ & G & $\mathrm{D}$ & $\mathrm{D}$ \\
\hline \multicolumn{2}{|l|}{ A/Wuxi/1/2013 } & $\mathrm{D}$ & $\mathrm{K}$ & $\mathrm{T}$ & $\mathrm{V}$ & I & A & I & $\mathrm{N}$ & $\mathrm{K}$ & G & $\mathrm{D}$ & $\mathrm{D}$ \\
\hline \multicolumn{2}{|l|}{ A/Wuxi/2/2013 } & $\mathrm{D}$ & K & $\mathrm{T}$ & $\mathrm{V}$ & I & A & I & $\mathrm{N}$ & $\mathrm{K}$ & G & $\mathrm{D}$ & $\mathrm{D}$ \\
\hline \multicolumn{2}{|c|}{ A/Environment/Wuxi/1/2013 } & $\mathrm{D}$ & K & $\mathrm{T}$ & $\mathrm{V}$ & I & A & I & $\mathrm{N}$ & E & G & $\mathrm{D}$ & $\mathrm{D}$ \\
\hline & \multicolumn{13}{|c|}{ PA } \\
\hline & $1-7$ & 70 & 86 & 100 & 206 & 263 & 272 & 308 & 394 & 400 & 409 & 621 & 716 \\
\hline A/Shanghai/1/2013 & No deletion & A & M & A & E & $\mathrm{T}$ & $\mathrm{N}$ & I & $\mathrm{N}$ & $\mathrm{P}$ & $\mathrm{N}$ & I & $\mathrm{R}$ \\
\hline A/Shanghai/2/2013 & No deletion & A & M & A & $\mathrm{E}$ & $\mathrm{T}$ & $\mathrm{N}$ & I & $\mathrm{N}$ & $\mathrm{P}$ & $\mathrm{N}$ & I & $\mathrm{R}$ \\
\hline A/Shanghai/3/2013 & No deletion & A & M & A & E & $\mathrm{T}$ & $\mathrm{N}$ & I & $\mathrm{N}$ & $\mathrm{P}$ & $\mathrm{N}$ & I & $\mathrm{R}$ \\
\hline A/Shanghai/4/2013 & No deletion & A & M & A & $\mathrm{E}$ & $\mathrm{T}$ & $\mathrm{N}$ & I & $\mathrm{N}$ & $\mathrm{P}$ & $\mathrm{N}$ & I & $\mathrm{R}$ \\
\hline A/Shanghai/4664T/2013 & No deletion & A & $\mathrm{V}$ & A & $\mathrm{V}$ & $\mathrm{T}$ & $\mathrm{N}$ & I & $\mathrm{N}$ & $\mathrm{P}$ & $\mathrm{N}$ & S & $\mathrm{K}$ \\
\hline $\begin{array}{l}\text { A/Chicken/shanghai/S105 } \\
\text { 3/2013 }\end{array}$ & No deletion & A & M & A & $\mathrm{E}$ & $\mathrm{T}$ & $\mathrm{N}$ & I & $\mathrm{N}$ & $\mathrm{P}$ & $\mathrm{N}$ & I & $\mathrm{R}$ \\
\hline $\begin{array}{c}\text { A/Pigeon/shanghai/S1069/ } \\
2013\end{array}$ & No deletion & A & M & A & $\mathrm{E}$ & $\mathrm{T}$ & $\mathrm{N}$ & I & $\mathrm{N}$ & $\mathrm{P}$ & $\mathrm{N}$ & I & $\mathrm{R}$ \\
\hline $\begin{array}{c}\text { A/Environment/shanghai/ } \\
\text { S1088/2013 }\end{array}$ & No deletion & A & M & A & $\mathrm{E}$ & $\mathrm{T}$ & $\mathrm{N}$ & I & $\mathrm{D}$ & $\mathrm{P}$ & $\mathrm{N}$ & I & $\mathrm{R}$ \\
\hline A/Zhejiang/01/2013 & No deletion & A & M & A & E & $\mathrm{T}$ & $\mathrm{N}$ & I & $\mathrm{N}$ & $\mathrm{P}$ & $\mathrm{N}$ & I & $\mathrm{R}$ \\
\hline A/Zhejiang/1/2013 & No deletion & A & M & A & $\mathrm{E}$ & $\mathrm{T}$ & $\mathrm{N}$ & I & $\mathrm{N}$ & $\mathrm{X}$ & $\mathrm{N}$ & I & $\mathrm{R}$ \\
\hline A/Zhejiang/2/2013 & No deletion & $\mathrm{V}$ & M & $\mathrm{V}$ & E & $\mathrm{T}$ & $\mathrm{D}$ & I & $\mathrm{D}$ & $\mathrm{X}$ & S & I & $\mathrm{R}$ \\
\hline $\begin{array}{l}\text { A/Zhejiang/DTID-ZJU01/ } \\
2013\end{array}$ & No deletion & $\mathrm{V}$ & M & $\mathrm{V}$ & $\mathrm{E}$ & $\mathrm{T}$ & $\mathrm{D}$ & I & $\mathrm{D}$ & $\mathrm{P}$ & $\mathrm{S}$ & I & $\mathrm{R}$ \\
\hline $\begin{array}{c}\text { A/Chicken/zhejiang/DTID } \\
\text {-ZJU01/2013 }\end{array}$ & Deletion & A & M & A & $\mathrm{E}$ & $\mathrm{T}$ & $\mathrm{N}$ & I & $\mathrm{N}$ & $\mathrm{P}$ & $\mathrm{N}$ & I & $\mathrm{R}$ \\
\hline A/Hangzhou/1/2013 & No deletion & A & M & A & $\mathrm{E}$ & $\mathrm{T}$ & $\mathrm{N}$ & I & $\mathrm{N}$ & $\mathrm{P}$ & $\mathrm{N}$ & I & $\mathrm{R}$ \\
\hline A/Hangzhou/2/2013 & No deletion & A & M & A & $\mathrm{E}$ & $\mathrm{T}$ & $\mathrm{N}$ & I & $\mathrm{N}$ & $\mathrm{P}$ & $\mathrm{N}$ & I & $\mathrm{R}$ \\
\hline A/Hangzhou/3/2013 & No deletion & A & M & A & $\mathrm{E}$ & A & $\mathrm{N}$ & $\mathrm{V}$ & $\mathrm{N}$ & $\mathrm{P}$ & $\mathrm{N}$ & I & $\mathrm{R}$ \\
\hline $\begin{array}{l}\text { A/Environment/Hangzhou } \\
\text { /34/2013 }\end{array}$ & No deletion & $\mathrm{A}$ & M & A & E & $\mathrm{T}$ & $\mathrm{N}$ & I & $\mathrm{N}$ & $\mathrm{P}$ & $\mathrm{N}$ & I & $\mathrm{R}$ \\
\hline A/Anhui/1/2013 & No deletion & A & M & A & $\mathrm{E}$ & $\mathrm{T}$ & $\mathrm{N}$ & I & $\mathrm{N}$ & $\mathrm{P}$ & $\mathrm{N}$ & I & $\mathrm{R}$ \\
\hline A/Wuxi/1/2013 & No deletion & A & M & A & $\mathrm{E}$ & $\mathrm{T}$ & $\mathrm{N}$ & I & $\mathrm{N}$ & $\mathrm{P}$ & $\mathrm{N}$ & I & $\mathrm{R}$ \\
\hline A/Wuxi/2/2013 & No deletion & A & M & A & $\mathrm{E}$ & $\mathrm{T}$ & $\mathrm{N}$ & I & $\mathrm{N}$ & $P$ & $\mathrm{~N}$ & I & $\mathrm{R}$ \\
\hline $\begin{array}{l}\text { A/Environment/Wuxi/1/ } \\
2013\end{array}$ & No deletion & A & M & A & E & $\mathrm{T}$ & $\mathrm{N}$ & $\mathrm{V}$ & $\mathrm{N}$ & $\mathrm{P}$ & $\mathrm{N}$ & I & $\mathrm{R}$ \\
\hline
\end{tabular}

Figure Labels: All polymerase gene sequences from H7N9 Influenza A virus strains available in the NCBI database were downloaded for alignment analysis. The amino acid mutations and sequence homology were determined and analyzed using the Meg Align H module of the DNASTAR package. Shaded boxes represent the functional domain of the polymerase protein. The numbers represent the differential amino acid site comparing 
between the H7N9 viruses.

\section{Acknowledgements}

The study was supported by grants from National Natural Science Foundation of China (Grant No. 31402216), the National Key Technologies Research and Development Program of China (2013ZX10004-610), China Ministry of Science and Technology (MOST) Project 973 (Grant No. 2011CB504705), Intramural special grant for influenza virus research from the Chinese Academy of Sciences (KJZD-EW-L09-2), the Key Research Program of the Chinese Academy of Sciences (KSZD-EW-Z-005-001). WJL is principal investigators of the NSFC Innovative Research Group (Grant No. 81321063).

\section{References}

[1] Chen, E., Chen, Y., Fu, L., Chen, Z., Gong, Z., Mao, H., Wang, D., Ni, M., Wu, P., Yu, Z., He, T., Li, Z., Gao, J., Liu, S., Shu, Y., Cowling, B., Xia, S. and Yu, H. (2013) Human Infection with Avian Influenza A(H7N9) Virus Re-Emerges in China in Winter 2013. Euro Surveillance, 18, pii: 20616.

[2] Dai, C. and Jiang, M. (2013) Understanding H7N9 Avian Flu. BMJ, 346, Article ID: f2755. http://dx.doi.org/10.1136/bmj.f2755

[3] Shi, B., Xia, S., Yang, G.J., Zhou, X.N. and Liu, J. (2013) Research Priorities in Modeling the Transmission Risks of H7N9 Bird Flu. Infectious Diseases of Poverty, 2, 8. http://dx.doi.org/10.1186/2049-9957-2-8

[4] Wu, Y. and Gao, G.F. (2013) Compiling of Comprehensive Data of Human Infections with Novel Influenza A (H7N9) Virus. Frontiers of Medicine, 7, 275-276.

[5] Xu, C., Havers, F., Wang, L., Chen, T., Shi, J., Wang, D., Yang, J., Yang, L., Widdowson, M.A. and Shu, Y. (2013) Monitoring Avian Influenza A(H7N9) Virus through National Influenza-Like Illness Surveillance, China. Emerging Infectious Diseases, 19, 1289-1292.

[6] Cowling, B.J., Jin, L., Lau, E.H., Liao, Q., Wu, P., Jiang, H., Tsang, T.K., Zheng, J., Fang, V.J., Chang, Z., Ni, M.Y., Zhang, Q., Ip, D.K., Yu, J., Li, Y., Wang, L., Tu, W., Meng, L., Wu, J.T., Luo, H., Li, Q., Shu, Y., Li, Z., Feng, Z., Yang, W., Wang, Y., Leung, G.M. and Yu, H. (2013) Comparative Epidemiology of Human Infections with Avian Influenza A H7N9 and H5N1 Viruses in China: A Population-Based Study of Laboratory-Confirmed Cases. The Lancet, 382, 129-137. http://dx.doi.org/10.1016/S0140-6736(13)61171-X

[7] Gao, R., Cao, B., Hu, Y., Feng, Z., Wang, D., Hu, W., Chen, J., Jie, Z., Qiu, H., Xu, K., Xu, X., Lu, H., Zhu, W., Gao, Z., Xiang, N., Shen, Y., He, Z., Gu, Y., Zhang, Z., Yang, Y., Zhao, X., Zhou, L., Li, X., Zou, S., Zhang, Y., Li, X., Yang, L., Guo, J., Dong, J., Li, Q., Dong, L., Zhu, Y., Bai, T., Wang, S., Hao, P., Yang, W., Zhang, Y., Han, J., Yu, H., Li, D., Gao, G.F., Wu, G., Wang, Y., Yuan, Z. and Shu, Y. (2013) Human Infection with a Novel Avian-Origin Influenza A (H7N9) Virus. The New England Journal of Medicine, 368, 1888-1897. http://dx.doi.org/10.1056/NEJMoa1304459

[8] Liu, D., Shi, W., Shi, Y., Wang, D., Xiao, H., Li, W., Bi, Y., Wu, Y., Li, X., Yan, J., Liu, W., Zhao, G., Yang, W., Wang, Y., Ma, J., Shu, Y., Lei, F. and Gao, G.F. (2013) Origin and Diversity of Novel Avian Influenza A H7N9 Viruses Causing Human Infection: Phylogenetic, Structural, and Coalescent Analyses. The Lancet, 381, 1926-1932. http://dx.doi.org/10.1016/S0140-6736(13)60938-1

[9] Kageyama, T., Fujisaki, S., Takashita, E., Xu, H., Yamada, S., Uchida, Y., Neumann, G., Saito, T., Kawaoka, Y. and Tashiro, M. (2013) Genetic Analysis of Novel Avian A(H7N9) Influenza Viruses Isolated from Patients in China. Euro Surveillance, 18, 20453.

[10] Zhang, Y., Zhang, Q., Gao, Y., He, X., Kong, H., Jiang, Y., Guan, Y., Xia, X., Shu, Y., Kawaoka, Y., Bu, Z. and Chen, H. (2012) Key Molecular Factors in Hemagglutinin and PB2 Contribute to Efficient Transmission of the 2009 H1N1 Pandemic Influenza Virus. Journal of Virology, 86, 9666-9674. http://dx.doi.org/10.1128/JVI.00958-12

[11] Hulse-Post, D.J., Franks, J., Boyd, K., Salomon, R., Hoffmann, E., Yen, H.L., Webby, R.J., Walker, D., Nguyen, T.D. and Webster, R.G. (2007) Molecular Changes in the Polymerase Genes (PA and PB1) Associated with High Pathogenicity of H5N1 Influenza Virus in Mallard Ducks. Journal of Virology, 81, 8515-8524. http://dx.doi.org/10.1128/JVI.00435-07

[12] Gao, Y., Zhang, Y., Shinya, K., Deng, G., Jiang, Y., Li, Z., Guan, Y., Tian, G., Li, Y., Shi, J., Liu, L., Zeng, X., Bu, Z., Xia, X., Kawaoka, Y. and Chen, H. (2009) Identification of Amino Acids in HA and PB2 Critical for the Transmission of H5N1 Avian Influenza Viruses in a Mammalian Host. PLoS Pathogens, 5, e1000709. http://dx.doi.org/10.1371/journal.ppat.1000709

[13] Hatta, M., Gao, P., Halfmann, P. and Kawaoka, Y. (2001) Molecular Basis for High Virulence of Hong Kong H5N1 Influenza A Viruses. Science, 293, 1840-1842. http://dx.doi.org/10.1126/science.1062882 
[14] Le, Q.M., Sakai-Tagawa, Y., Ozawa, M., Ito, M. and Kawaoka, Y. (2009) Selection of H5N1 Influenza Virus PB2 during Replication in Humans. Journal of Virology, 83, 5278-5281. http://dx.doi.org/10.1128/JVI.00063-09

[15] Steel, J., Lowen, A.C., Mubareka, S. and Palese, P. (2009) Transmission of Influenza Virus in a Mammalian Host Is Increased by PB2 Amino Acids 627K or 627E/701N. PLoS Pathogens, 5, e1000252. http://dx.doi.org/10.1371/journal.ppat.1000252

[16] Gonzalez, S. and Ortin, J. (1999) Distinct Regions of Influenza Virus PB1 Polymerase Subunit Recognize vRNA and cRNA Templates. The EMBO Journal, 18, 3767-3775. http://dx.doi.org/10.1093/emboj/18.13.3767

[17] Gonzalez, S. and Ortin, J. (1999) Characterization of the Influenza Virus PB1 Protein Binding to Viral RNA: Two Separate Regions of the Protein Contribute to the Interaction Domain. Journal of Virology, 73, 631-637.

[18] Chu, C., Fan, S., Li, C., Macken, C., Kim, J.H., Hatta, M., Neumann, G. and Kawaoka, Y. (2012) Functional Analysis of Conserved Motifs in Influenza Virus PB1 Protein. PLoS ONE, 7, e36113. http://dx.doi.org/10.1371/journal.pone.0036113

[19] Perez, D.R. and Donis, R.O. (2001) Functional Analysis of PA Binding by Influenza A Virus PB1: Effects on Polymerase Activity and Viral Infectivity. Journal of Virology, 75, 8127-8136. http://dx.doi.org/10.1128/JVI.75.17.8127-8136.2001

[20] Zamarin, D., Ortigoza, M.B. and Palese, P. (2006) Influenza A Virus PB1-F2 Protein Contributes to Viral Pathogenesis in Mice. Journal of Virology, 80, 7976-7983. http://dx.doi.org/10.1128/JVI.00415-06

[21] Herfst, S., Schrauwen, E.J., Linster, M., Chutinimitkul, S., de Wit, E., Munster, V.J., Sorrell, E.M., Bestebroer, T.M., Burke, D.F., Smith, D.J., Rimmelzwaan, G.F., Osterhaus, A.D. and Fouchier, R.A. (2012) Airborne Transmission of Influenza A/H5N1 Virus between Ferrets. Science, 336, 1534-1541. http://dx.doi.org/10.1126/science.1213362

[22] de Wit, E., Munster, V.J., van Riel, D., Beyer, W.E., Rimmelzwaan, G.F., Kuiken, T., Osterhaus, A.D. and Fouchier, R.A. (2010) Molecular Determinants of Adaptation of Highly Pathogenic Avian Influenza H7N7 Viruses to Efficient Replication in the Human Host. Journal of Virology, 84, 1597-1606. http://dx.doi.org/10.1128/JVI.01783-09

[23] Desmet, E.A., Bussey, K.A., Stone, R. and Takimoto, T. (2013) Identification of the N-Terminal Domain of the Influenza Virus PA Responsible for the Suppression of Host Protein Synthesis. Journal of Virology, 87, 3108-3118. http://dx.doi.org/10.1128/JVI.02826-12

[24] Hemerka, J.N., Wang, D., Weng, Y., Lu, W., Kaushik, R.S., Jin, J., Harmon, A.F. and Li, F. (2009) Detection and Characterization of Influenza A Virus PA-PB2 Interaction through a Bimolecular Fluorescence Complementation Assay. Journal of Virology, 83, 3944-3955. http://dx.doi.org/10.1128/JVI.02300-08

[25] Song, M.S., Pascua, P.N., Lee, J.H., Baek, Y.H., Park, K.J., Kwon, H.I., Park, S.J., Kim, C.J., Kim, H., Webby, R.J., Webster, R.G. and Choi, Y.K. (2011) Virulence and Genetic Compatibility of Polymerase Reassortant Viruses Derived from the Pandemic (H1N1) 2009 Influenza Virus and Circulating Influenza A Viruses. Journal of Virology, 85, 62756286. http://dx.doi.org/10.1128/JVI.02125-10

[26] Qi, X., Qian, Y.H., Bao, C.J., Guo, X.L., Cui, L.B., Tang, F.Y., Ji, H., Huang, Y., Cai, P.Q., Lu, B., Xu, K., Shi, C., Zhu, F.C., Zhou, M.H. and Wang, H. (2013) Probable Person to Person Transmission of Novel Avian Influenza A (H7N9) Virus in Eastern China, 2013: Epidemiological Investigation. British Medical Journal, 347, Article ID: f4752. http://dx.doi.org/10.1136/bmj.f4752

[27] Wu, A., Su, C., Wang, D., Peng, Y., Liu, M., Hua, S., Li, T., Gao, G.F., Tang, H., Chen, J., Liu, X., Shu, Y., Peng, D., and Jiang, T.J. (2013) Sequential Reassortments Underlie Diverse Influenza H7N9 Genotypes in China. Cell Host \& Microbe, 14, 446-452. http://dx.doi.org/10.1016/j.chom.2013.09.001

[28] Plotkin, J.B. and Dushoff, J. (2003) Codon Bias and Frequency-Dependent Selection on the Hemagglutinin Epitopes of Influenza A Virus. Proceedings of the National Academy of Sciences of the United States of America, 100, 7152-7157. http://dx.doi.org/10.1073/pnas.1132114100

[29] The Lancet Infectious Diseases (2013) A Proportionate Response to H7N9. The Lancet Infectious Diseases, 13, 465. http://dx.doi.org/10.1016/S1473-3099(13)70134-8

[30] Naffakh, N., Massin, P., Escriou, N., Crescenzo-Chaigne, B. and van der Werf, S. (2000) Genetic Analysis of the Compatibility between Polymerase Proteins from Human and Avian Strains of Influenza A Viruses. Journal of General Virology, 81, 1283-1291. 